\title{
Mycobacterium chimaera: The ethical duty to disclose the minimal risk of infection to exposed patients
}

\author{
Anita Nguyen, MBBS, ${ }^{a}$ C. Christopher Hook, MD, ${ }^{b}$ Joseph A. Dearani, MD, ${ }^{a}$ and Hartzell V. Schaff, MD $^{a}$
}

\footnotetext{
From the ${ }^{\mathrm{a}}$ Department of Cardiovascular Surgery and ${ }^{\mathrm{b}}$ Division of Hematology, Mayo Clinic, Rochester, Minn. This work was supported by the Paul and Ruby Tsai Family.

Disclosures: Authors have nothing to disclose with regard to commercial support

Anita Nguyen is a postdoctoral research fellow in the Department of Cardiovascular Surgery at Mayo Clinic. C. Christopher Hook is Associate Professor of Medicine at Mayo Clinic College of Medicine and Science and Consultant with the Division of Hematology, was founder of Mayo Clinic Ethics Consultation Services in Jacksonville, Florida, and Rochester, Minnesota, and the Mayo Clinical Ethics Council. He currently serves as the chair of Ethics Education in the Mayo Enterprise Office of Ethics. Dr Hook was a cardiac surgery patient in June 2016 and is a recipient of the M chimaera notification letter discussed in this article. Joseph A. Dearani is a Professor of Surgery at Mayo Clinic College of Medicine and Science and Chair of the Department of Cardiovascular Surgery at Mayo Clinic. He has led Mayo Clinic's institutional response following the discovery of $M$ chimaera in certain heater-cooler units used during cardiac surgery. Hartzell V. Schaff is a Consultant in the Department of Cardiovascular Surgery and Stuart W. Harrington Professor of Surgery at Mayo Clinic.

Received for publication Dec 15, 2016; revisions received Jan 24, 2017; accepted for publication Feb 10, 2017; available ahead of print March 25, 2016.

Address for reprints: Hartzell V. Schaff, MD, Department of Cardiovascular Surgery, Mayo Clinic, 200 First St SW, Rochester, MN 55905 (E-mail: schaff@mayo.edu).

J Thorac Cardiovasc Surg 2017;153:1422-4

$0022-5223 / \$ 36.00$

Copyright (C) 2017 by The American Association for Thoracic Surgery

http://dx.doi.org/10.1016/j.jtcvs.2017.02.020
}

A recent study by Sommerstein and colleagues ${ }^{1}$ has demonstrated a causal link between Mycobacterium chimaera infection and the LivaNova PLC Stöckert 3T heater-cooler system (LivaNova PLC, London, United Kingdom) used in cardiac surgery. The heater-cooler unit disrupts laminar air flow and enables airborne spread of $M$ chimaera from a contaminated unit to a surgical site. $M$ chimaera infection may not manifest immediately, and infections occurring up to 4 years after cardiac surgery have been reported. ${ }^{2,3}$ These cases are rare, and the largest series from a single institution includes 6 patients over an experience of 3700 operations during a 4-year period (a cross-sectional prevalence of $0.16 \%$ ). ${ }^{2}$ Nevertheless, $M$ chimaera infection can cause serious complications, including prosthetic valve endocarditis, aortic graft infection, and myocarditis. ${ }^{2}$ The initial diagnostic workup of a patient with suspected $M$ chimaera infection consists of acid-fast bacilli smear and culture. Definitive diagnosis may require more specific tests such as the BACTEC Myco/F Lytic culture vial (Becton, Dickinson and Company, Franklin Lakes, NJ) for blood culture, which does not yield prompt results and is not widely available. There is no consensus regarding optimal antimicrobial therapy, and combinations of clarithromycin, rifabutin, ethambutol, and amikacin have been used. ${ }^{2}$ Mortality from $M$ chimaera infection is high, which is mainly due to nonspecific presentation and late diagnosis, as well as the challenging nature of finding adequate antimicrobial therapy.

In light of this evidence, the US Food and Drug Administration (FDA) issued recommendations on June 1, 2016, advising health care facilities and staff to "determine a method for patient follow-up and establish patient situation.

\section{AUTONOMY}

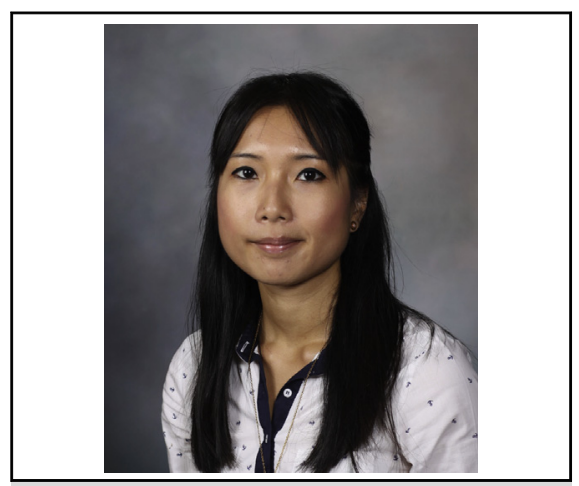

Anita Nguyen, MBBS

Central Message

There is an ethical duty to disclose the minimal risk of Mycobacterium chimaera infection to patients who were potentially exposed during cardiac surgery.

See Editorial Commentary page 1425 .

surveillance in cases of potential exposure." ${ }^{5}$ On October 13, 2016, an official health advisory from the Centers for Disease Control and Prevention (CDC) stated that "hospitals [are advised] to notify patients who underwent open surgery involving a Stöckert 3T heater-cooler that the device was potentially contaminated, possibly putting patients at risk for a life threatening infection." 6 Notification as recommended but not mandated in these guidelines likely will cause anxiety to a large number of postoperative patients. In the United States, the responsibility lies with individual cardiac surgery centers to address this issue, and some clinicians may question the dilemma of transparency versus raising unnecessary concern as they determine the best way to handle this

As we examined the current literature on $M$ chimaera infection and the logistic challenges that implementing these guidelines pose, we addressed whether there is an ethical duty of disclosing this information to patients considering the 4 principles of medical ethics: autonomy, beneficence, nonmaleficence, and justice.

Current ethical principles favor full disclosure of all relevant information to patients out of respect for patient autonomy. The only situation in which nondisclosure is 
considered ethical is if a patient specifically asks for information to be withheld. ${ }^{8,9}$

A common clinical scenario in which detailed disclosure is required is when discussing the risks of a surgical procedure. It is standard practice to explain the minimal but serious risks of a surgical procedure to a patient when obtaining consent. A patient can only make a truly informed decision on whether to pursue surgical treatment if he or she is equipped with all relevant information. The onus is then on the patient to decide how to use this information, and this forms the basis of the principle of respect for patient autonomy.

However, the challenges of disclosing information that may not be deemed clinically significant are exemplified in another field of medicine: genetics. Genetic testing is conducted to identify genes that leave the patient susceptible to developing specific diseases. Issues concerning disclosure can arise with incidental findings. Although no standard guidelines have been set regarding the disclosure of incidental findings, current opinion favors full disclosure of incidental findings that have the potential of causing disease, whereas disclosure of incidental findings of uncertain significance is not deemed necessary. ${ }^{10-12}$

Although $M$ chimaera infection occurs rarely after cardiac surgery, there are serious risks and complications associated with the infection. For this reason, full disclosure of the potential exposure to M chimaera is consistent with current ethical thinking regarding respect for patient autonomy.

\section{BENEFICENCE AND NONMALEFICENCE}

Beneficence means acting in the best interest of the patient, and nonmaleficence is the principle that dictates physicians do no harm or should minimize unavoidable harm in diagnosis and treatment. In the past, the principles of beneficence and nonmaleficence have been used to justify nondisclosure of information to patients, for example, using the argument of therapeutic privilege. In such cases, withholding information from the patient to prevent harm or distress was thought to be in the patient's best interest. However, this approach to medical disclosure would now be considered paternalistic and is only acceptable in exceptional circumstances, in which the patient will, with high likelihood, engage in self-injurious behavior upon learning the information.

In the case of $M$ chimaera, the risk of infection is minimal; however, the consequences are potentially devastating. Disclosure of this information enables patients to be more vigilant about symptoms and to seek medical advice. This could increase the chances of infection being detected at an earlier stage, when it might be more amenable to treatment. Nondisclosure of risks of $M$ chimaera infection has the potential of causing serious harm to a small number of patients and would be considered a breach of the principles of beneficence and nonmaleficence.

\section{JUSTICE}

The last ethical principle to consider is justice, which entails the fair and just treatment of all patients. A commonly discussed example concerning the principle of justice is the hypothetical scenario of an influenza pandemic. In this case, the CDC advocates attempting fair resource allocation without violating any of the other principles of medical ethics. However, in practical terms, the CDC recommends prioritizing treatment of people who are "key to the preservation of society." 13 Equal distribution of limited resources would be unfeasible in a global outbreak of a communicable disease, and priorities regarding resource allocation would have to be set.

However, M chimaera infection could be considered the complete opposite of an influenza pandemic. This infection occurs rarely, and the risk of acquiring $M$ chimaera after cardiac surgery is less than $0.2 \%{ }^{2}$ Furthermore, the disease is not communicable. Thus, although the number of patients who could have been exposed to $M$ chimaera is high, the number of patients who will likely acquire the infection is extremely low. Fair distribution of resources should be achievable by focusing on informing patients of the risk of $M$ chimaera infection and limiting further consultation and testing to patients with worrisome symptoms, which is in line with CDC and FDA recommendations. ${ }^{14}$

\section{PRACTICAL CONSIDERATIONS: THE MAYO CLINIC EXPERIENCE}

At our institution, the recommendations of the CDC and FDA prompted a rapid and substantial response; a crisis management team was assembled, and within days of publication of the guidelines, we identified more than 17,000 patients who underwent cardiac surgery at 1 of 4 sites (Mayo Clinic in Rochester, Minn, Phoenix, Ariz, and Jacksonville, Fla, and Mayo Clinic Health System-Eau Claire Hospital in Eau Claire, Wis) within the last 5 years. We sent letters to all of these patients, advising them of the recently discovered risk of $M$ chimaera infection (Appendices 1 and 2). These letters were translated into the patient's first language and sent to national and international addresses by certified mail. When letters did not reach the addressee, a second attempt was made to ensure as many patients as possible would receive our communication. In our correspondence, we stressed the extremely low chances of infection and suggested that patients be alert to symptoms of ongoing night sweats, wound infection, unintentional weight loss, and unexplained fever. We also implemented a telephone triage line staffed by trained nurses to be a resource for our patients. Calls that could not be resolved via the triage 
line were escalated to nurse practitioners or physician's assistants, and consultants. So far, we have received up to 50 calls per day from concerned patients, mostly to clarify questions but also to express gratitude about our prompt correspondence. Only 2 patients had symptoms that consultants deemed concerning enough to be referred back to Mayo Clinic for further testing.

At the time of notification, 10 Stöckert $3 \mathrm{~T}$ heater-cooler systems were in use at Mayo Clinic in Rochester, Minnesota. These devices were discontinued when cultures from the units became positive for M chimaera; all of these heater-coolers tested positive for $M$ chimaera and were replaced with alternative devices (Sarns Dual Heater Cooler Model 11160; Terumo, Tokyo, Japan).

We think there is an ethical duty to disclose the minimal risk of $M$ chimaera to patients who have had potential exposure. We suggest that, at minimum, patients should receive written communications stressing the extremely small risk of developing infection after cardiac surgery. Additional sources of reliable information (eg, CDC and FDA Web sites and telephone numbers) and contact details also should be provided to patients. Only patients with truly worrisome symptoms, in whom a high clinical suspicion for an atypical infection exists and common causes of infection have been excluded, should receive further testing, as advised by the CDC and FDA. ${ }^{14}$

\section{LEGAL CONSIDERATIONS}

The possibility of litigation is ever present in the United States, and we have been made aware of law firms contacting patients to discuss potential class action lawsuits. Furthermore, a quick investigation on search engines using the terms "mycobacterium chimaera" and "class action lawsuit" yields multiple results of law firms offering free consultations to exposed patients. Although not a primary motivation, the possibility of litigation should further warrant prompt disclosure of the risk of $M$ chimaera infection to patients with potential exposure and a plan to address the concerns of these patients.

\section{CONCLUSIONS}

We believe that there is an ethical duty to disclose the minimal risk of $M$ chimaera infection to all patients with potential exposure to the organism during cardiac surgery. Although we acknowledge the risk of causing unnecessary distress in a large patient population, the ethical duty of disclosure takes precedence, and we advocate prompt communication of the potential hazard of infection with exposed patients.

\section{References}

1. Sommerstein R, Rüegg C, Kohler P, Bloemberg G, Kuster SP, Sax H. Transmission of Mycobacterium chimaera from heater-cooler units during cardiac surgery despite an ultraclean air ventilation system. Emerg Infect Dis. 2016;22:1008-13

2. Kohler P, Kuster SP, Bloemberg G, Schulthess B, Frank M, Tanner FC, et al. Healthcare-associated prosthetic heart valve, aortic vascular graft, and disseminated Mycobacterium chimaera infections subsequent to open heart surgery. Eur Heart J. 2015;36:2745-53.

3. Sax H, Bloemberg G, Hasse B, Sommerstein R, Kohler P, Achermann Y, et al. Prolonged outbreak of Mycobacterium chimaera infection after open-chest heart surgery. Clin Infect Dis. 2015;61:67-75.

4. Achermann Y, Rössle M, Hoffmann M, Deggim V, Kuster S, Zimmermann DR, et al. Prosthetic valve endocarditis and bloodstream infection due to Mycobacterium chimaera. J Clin Microbiol. 2013;51:1769-73.

5. US Food and Drug Administration. Mycobacterium chimaera infections associated with Sorin Group Deutschland GmBH Stöckert 3T Heater-Cooler System: FDA Safety Communication. June 1, 2016. Washington, DC: US Department of Health and Human Services. FDA Med Bull. 2016.

6. Centers for Disease Control and Infection. CDC advises hospitals to alert patients at risk from contaminated heater-cooler devices used during cardiac surgery. Available at: https://emergency.cdc.gov/han/han00397.asp. Accessed November $30,2016$.

7. Beauchamp TL, Childress JF. Principles of Medical Ethics. New York: Oxford University Press; 1994.

8. Murray B. Informed consent: what must a physician disclose to a patient? Virtual Mentor. 2012;14:563.

9. Snyder L. American College of Physicians Ethics, Professionalism, and Human Rights Committee. American College of Physicians Ethics Manual: 6th ed. Ann Intern Med. 2012;156(1 Pt 2):73-104.

10. Gliwa C, Berkman BE. Do researchers have an obligation to actively look for genetic incidental findings? Am J Bioeth. 2013;13:32-42.

11. Weiner C. Anticipate and communicate: ethical management of incidental and secondary findings in the clinical, research, and direct-to-consumer contexts (December 2013 report of the Presidential Commission for the Study of Bioethical Issues. Am J Epidemiol. 2014;180:562-4.

12. Hofmann B. Incidental findings of uncertain significance: To know or not to know-that is not the question. BMC Med Ethics. 2016;17:13.

13. Holloway R, Rasmussen SA, Zaza S, Cox NJ, Jernigan DB. Updated preparedness and response framework for influenza pandemics. MMWR Recomm Rep. 2014;63(RR-06):1-18.

14. US Food and Drug Administration. Update: Mycobacterium chimaera infections associated with LivaNova PLC (formerly Sorin Group Deutschland GmBH) Stöckert 3T Heater-Cooler System: FDA Safety Communication. October 13, 2016. Washington, DC: US Department of Health and Human Services. FDA Med Bull. 2016. 


\section{APPENDIX E1. PATIENT NOTIFICATION LETTER: ADULT VERSION}

Dear [Patient Name],

Patient safety is Mayo Clinic's highest priority. The Mayo Clinic recently was made aware of a potential infection risk related to a device used at Mayo Clinic and at hospitals throughout the world during open surgeries. The CDC and FDA have investigated reports of a specific type of rare infection within the United States linked to the manufacturing process for this device, which is used to heat and cool a patient's blood during surgery.

The Mayo Clinic is taking steps as recommended by the $\mathrm{CDC}$ to minimize infection risk to patients. According to our records, you had an open surgery that may have exposed you to this infection. This letter is to inform you and other patients who have had open surgery of this risk and to provide some basic information about this specific infection.

The device has been linked to a rare infection caused by a type of bacteria known as "nontuberculous mycobacterium." There are several important things for you to know about this type of infection.

- Your chances of getting this infection are very low. The risk of getting this infection during open surgery is estimated to be less than $1 \%$.

- The infection is very slow growing.

- Symptoms of the infection typically occur within 22 months of the surgery.

- Symptoms include ongoing night sweats, wound infection, weight loss greater than $5 \%$ of your usual body weight over a period of several months, and unexplained fever with a temperature higher than $100.9^{\circ} \mathrm{F}\left(38.3^{\circ} \mathrm{C}\right)$ measured on more than 1 occasion.

- The infection cannot be spread from person to person.

- There are steps you can take if you have symptoms.

Your Mayo Clinic care team strongly encourages you to discuss any symptoms or questions with your primary care provider. In addition, please contact our Ask Mayo Clinic nurse line with questions at 1-800-951-3836 within the United States or 1-507-538-5764 if calling internationally.

Thank you for partnering with us to be watchful for symptoms and for staying in contact with your care team if you have concerns.

Sincerely,

Executive Dean of Practice

\section{APPENDIX E2. PATIENT NOTIFICATION LETTER: PEDIATRIC VERSION}

Dear [Parent Name],

Patient safety is Mayo Clinic's highest priority. The Mayo Clinic recently was made aware of a potential infection risk related to a device used at Mayo Clinic and at hospitals throughout the world during open surgeries. The CDC and FDA have investigated reports of a specific type of rare infection linked to the manufacturing process for this device, which is used to heat and cool a patient's blood during surgery.

The Mayo Clinic is taking steps as recommended by the $\mathrm{CDC}$ to minimize infection risk to patients. According to our records, your child had an open surgery, which may have exposed your child to this infection. This letter is to inform you and other parents whose children have had open surgery of this risk and to provide some basic information about this specific infection.

The device has been linked to a rare infection caused by a type of bacteria known as "nontuberculous mycobacterium." As a parent, there are several important things for you to know about your child's risk of this type of infection.

- Your child's chances of getting this infection are very low. The risk of getting this infection during open surgery is estimated to be less than $1 \%$.

- The infection is very slow growing.

- Symptoms of the infection typically occur within 22 months of the surgery.

- Symptoms include ongoing night sweats, wound infection, poor weight gain or weight loss, and unexplained fever with a temperature higher than $100.9^{\circ} \mathrm{F}\left(38.3^{\circ} \mathrm{C}\right)$ measured on more than 1 occasion.

- The infection cannot be spread from person to person.

- There are steps you can take if your child has symptoms.

Your Mayo Clinic care team strongly encourages you to discuss any symptoms or questions with your child's primary care provider. In addition, please contact our Ask Mayo Clinic nurse line with questions at 1-800-951-3836 within the United States or 1-507-538-5764 if calling internationally.

Thank you for partnering with us to be vigilant to watch your child for symptoms and for staying in contact with your child's care team if you have concerns.

Sincerely,

Executive Dean of Practice 